\title{
PROSPECTS FOR THE DETECTION OF MICROLENSING TIME DELAYS
}

\author{
CHRISTOPHER B. MOORE AND JACQUELINE N. HEWITT \\ Massachusetts Institute of Technology Cambridge, MA, USA
}

\begin{abstract}
Since the image separations produced by microlensing are inaccessible to observation, we are left to observe either a change in total amplitude or the time delay between the images. Spillar (1993) has suggested that a time delay might be observed in the autocorrelation of a microlensed signal. The time delays are of order a few microseconds and are easily accessible to a sufficiently wide bandwidth system. We calculate expected observational results using the Green Bank Telescope with a VLBA recording system, and find that brightness temperatures exceeding the Compton limit are required for detection.
\end{abstract}

\section{Discussion}

We consider microlensing of a background source (e.g. an AGN) by a single Schwarzschild potential in some intervening galaxy (or galaxy halo). In what follows, we take $q_{0}=0.5, H_{0}=80 \mathrm{kms}^{-1} \mathrm{Mpc}^{-1}, z_{s}=1.0, z_{l}=0.05$, lens mass $=\mathrm{M}_{\odot}$, and a uniform disc source of flux density $100 \mathrm{mJy}$. For a point source lensed by a single Schwarzschild potential the observed electric field is given in terms of the field that would be observed if there were no lens, $E(t)$. The observed field consists of two parts, one for each image: $\sqrt{\mu_{1}} E(t)+\sqrt{\mu_{2}} E(t+\Delta)$ where $\Delta$ is the gravitational lens time delay and $\mu_{1,2}$ are the magnification factors for the two images. The autocorrelation of this signal has three terms, the first of which appears at $\rho=0$ and corresponds to the total power of the two images added together. The second two terms appear at $\rho= \pm \Delta$ and allow us to measure the gravitational time delay and $\sqrt{\mu_{1} \mu_{2}}$. When observing with limited bandwidth, the observed autocorrelation is convolved with the autocorrelation of the impulse 
response function of the bandpass filter. For the VLBA $16 \mathrm{MHz}$ bandpass, the first zero occurs at a lag of $3.5 \times 10^{-8} \mathrm{sec}$.

If the source is not point-like and different regions radiate incoherently, then one can divide the source into a large number of point sources and sum their contributions to the autocorrelation signal. We have written software which generates a predicted autocorrelation function from a distribution of flux density on the sky, a bandpass, and the relevant lens parameters.

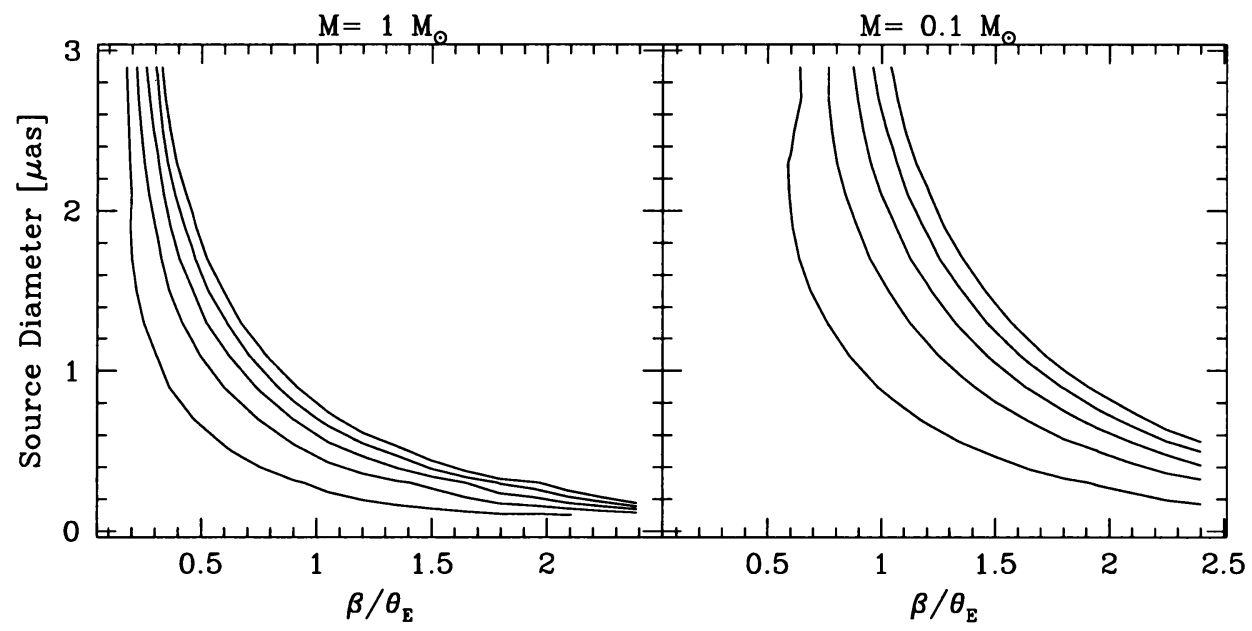

Figures 1 and 2 (above) show the observation time necessary to obtain a $3 \sigma$ single channel detection for various combinations of source size, lens to source distance, and lens mass. The contours are at intervals of two hours starting at 1 hour. Detection in less than 10 hours observation time requires sources with apparent $T_{B} \sim 10^{15} \mathrm{~K}$ which exceeds the Compton limit. Such high brightness temperatures are suggested by studies of intraday variability in AGN which report $T_{B}$ as high as $10^{19} \mathrm{~K}$ inferred from variability (Quirrenbach et al. 1992).

\section{Conclusions}

We have shown that microlensing time delays should be visible with large telescopes if there are high apparent $T_{B}$ sources and a sufficient mass density of lensing objects. Remaining to be considered explicitly are the effects of scintillation, the effect of multiple lensing events on detectability, and the special case of a lens with a diffuse object directly behind it.

\section{References}

Quirrenbach, A., Witzel, A., Krichbaum, T., Hummel, C., Wegner, R., Schalinski, C., Ott, M., Alberdi, A., \& Rioja, M., 1992, A\&A, 258, 279

Spillar, E., 1993, ApJ, 403, 20 